\title{
Dispositif d'amortissement de la veine pour les déchargeurs de Fessenheim
}

\section{Damping device in the by-pass channels at Fessenheim}

\author{
P.M (i. BRAT'DEATI"
}

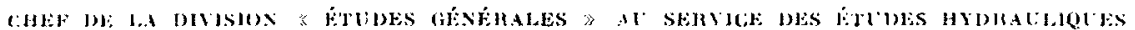

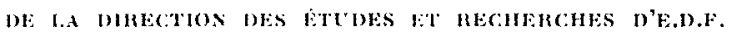

\begin{abstract}
Apris aboir silué le problème des déchargs'urs pour des asines en série sur un grand canal do dérivation, on décrit un. dispositif brise-charge composé d'une grille épaisse - mis au point pour l'usine de Fessenheim. Le fonctionnement en est analysé. On insiste sur un anantage important: sa grande souplesse d'adaptation susceptible de rendre son emploi assez général.
\end{abstract}

\begin{abstract}
After the problem of by-pass chunnels for power slations in series is established for " large deviation canal, an energy dissipating device composed of a thick grill is describeddeveloped for Fessenheim power station. The operation is artalysed. An important advantage is insisted upon; its great flexibility which is likely to make it more generally used.
\end{abstract}

I

L'aménagement des basses chutes à gros débits, placées sur de grands canaux de dérivation souvent utilisés par la navigation, exige que l'on assure la continuité de l'écoulement du débit disponible en cas de déclenchement ou d'arrèt d'un ou de plusieurs groupes.

C'est là un problème de vannes et d'asservissement de leur commande.

Il est également important qu'un fonctionnement prolongé ne menace pas la stabilité des ouvrages et du canal, et que l'écoulemenl aval ne rende pas inacceptables les conditions de navigation à proximitè des sorties d'écluses.

Un tel fonctionnement prolongé des déchargeurs est à envisager lorsque plusieurs chutes sont équipées en série sur le même canal, l'ensemble des usines de la chainc ne devant pas souffrir de la défaillance particlle ou totale de l'une d'entre elles. C'est le cas du Grand Can:I d'Alsace.

II

Un déchargeur évacue sous la mème chute le mème débit que la turbine à laquelle il est ad- joint. L'énergie de l'eau se trouve done entièrement disponible á la sorlic des vannes, sous forme cincétique pour la plus grande partie. Un ressaut, fixé par ies conditions aval, en transformera un peu en énergie de position, au prix d'une certaine perte. De ce qui reste, une partie se dissipera par frottements aux parois, risquant de eréer de gros dommages aux berges et au fond du canal, une autre restera sous forme cinétique et pourra gêner fortement la navigation si elle se traduit par la formation de courants secondaires diffusant lentement, avec gradients de vitesses importants et accompagnés de perturbation de surface et de tourbillons à grande échelle peu souhaitables.

III

Line solurtion simple consiste à fractionner suffisamment les débits de décharge en les étalant sur le maximum de largeur du canal. La dissipation d'énergic par frottement et turbulence peut alors se produire sans dommage important, les efforts étant répartis sur le maximum de surface de paroi et aucun courant secondaire à gros débil et grande vitesse ne pouvant se former. 
Ce parti fut adopté à Kembs, puis à Ottmarsheim, avec des résultals tout a fait acceptables.

Toutefois, celle solution conduit à une disposition d'ouvrages présentant quelques sujections. notamment une certaine complexité du génie civil et l'importance de la machinerie.

\section{IV}

Pour Fessenheim, la Région d'Equipement Hydraulique Nord a donc décidé d'étudier la possibilité dévacuer les débits de décharge par quatre pertuis reclilignes accolés, dans laxe de la cenIrale. Chacun serait capable de $300 \mathrm{~m}^{3} / \mathrm{s}$, c'esta-dire sensiblement le débit de l'un des 4 groupes constituant l'usine.

Bien qu'allongeant légèrement l'ensemble de lusine, une telle solution conduisait à une synetrie ef à une simplieite de bon aloi. Elle sem- hla devoir ètre adoptée sous réserve qu'un fonctionnement hydraulique correct en fìt mis au point.

L'étude fut confice au Laboratoire de Chatou. On décida de réaliser une usine à quatre groupes, comportant dans son axe deux groupes de deux pertuis, séparés par un mur suideau. Dans chacun de ces deux groupes. les veines liquides se rejoignent immédiatement après les rannes sur un coursier unique, au point bas duguel fut disposí un brise-charge d'un type assez. particulier.

\section{V}

Rappelons que Fessenheim est équipé pour $1.160 \mathrm{~m}^{3} / \mathrm{s}$ sous $15 \mathrm{~m}$.

Les cotes essentielles sont indiquées sur les figures 1 et 2. Notons que la forte contre-pente

\section{lini. 1.}

Indele an 1 2. profil réalise sur le modele.
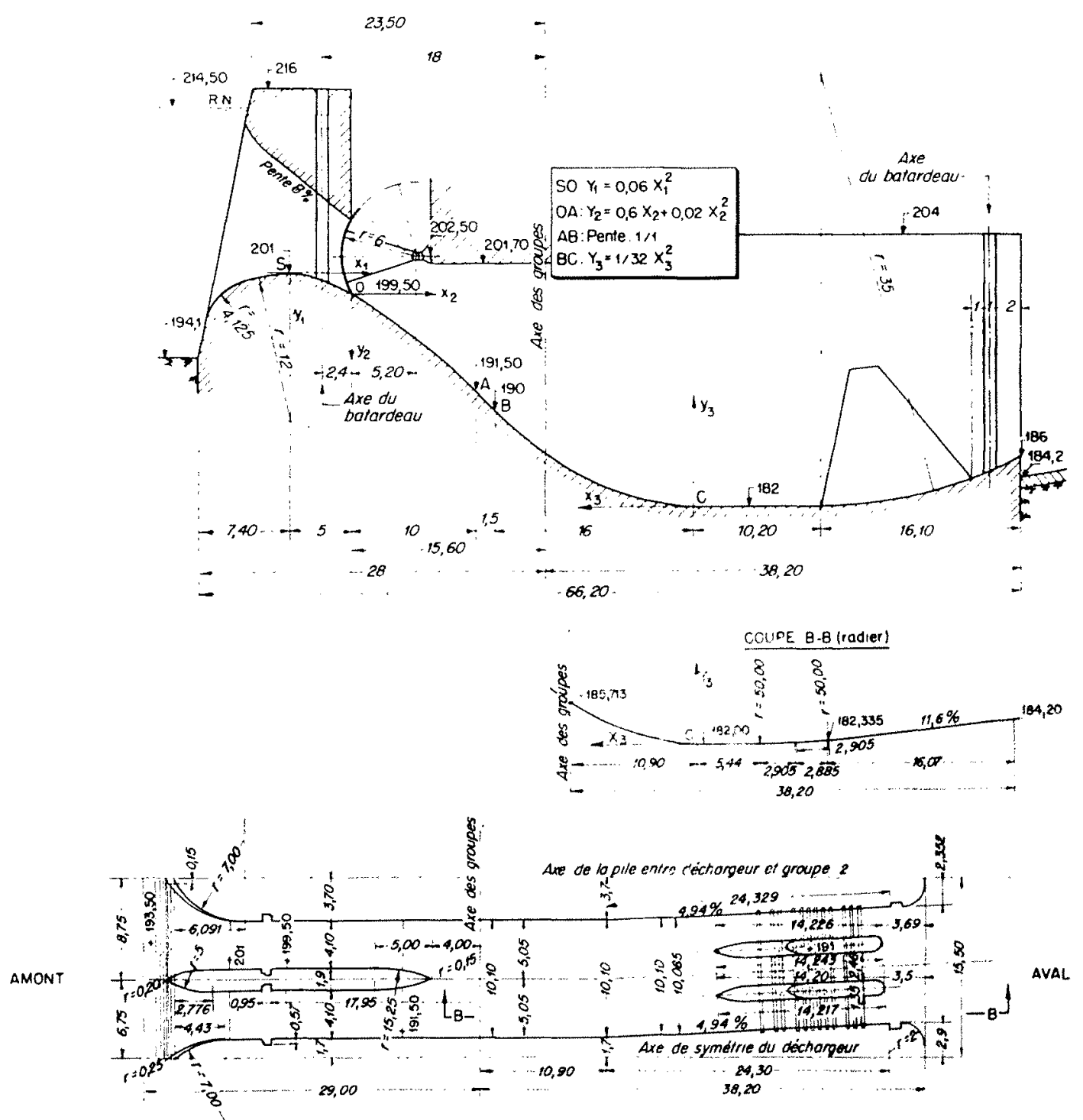

Fili. 2. Vue en plan du dednargene hedi. 
du radier (16\%), imposée par la traversée du rideau d'ćlanchéité aval, constituait une menace d'affouillements importants. Elle rendit plus dilicate la mise au point du brise-charge.

\section{VI}

La décharge dans le bief aval d'un díbil de cette importance, à une vitesse de 10 ì $12 \mathrm{~m} / \mathrm{s}$. en effet sur le ressaut et sur l'écoulement aval, en changeant le nombre et la disposition des barreaux, et aussi dans une certaine mesure en déplaçant parallèlement à l'axe la position du brise-charge.

Il est inutile de détailler l'ensemble des essais. C'est évidemment une méthode d'approximations successives qui les guida. Les critères adoptés ćtaient la forme des répartitions de vitesse aval, la stabililé du rouleau à axe horizonta! consti-
Fini, 3.

Modele au $1 / 25$. Disposition $n^{\prime \prime} 4$.

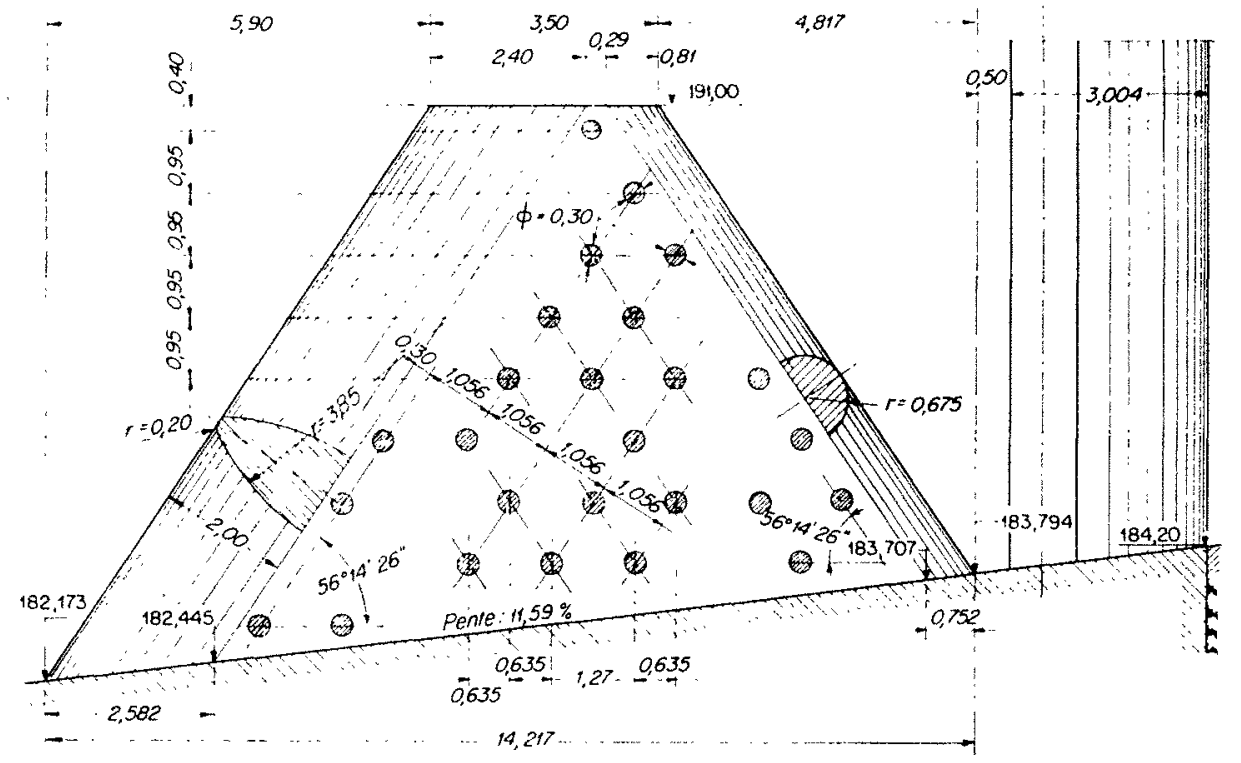

obligeait soit à donner au radier aval une résislance considérable en le prolongeant d'ailleurs assez loin, soit à localiser le ressaut à l'intérieur. des passes. à le stabiliser, toul en imposant a l'écoulement lluvial une répartition des vitesses qui ménage les fonds sans présenter pour autanl de maximum marqué.

Pour réaliser ces conditions, nous avons disposé au point bas des passes un brise-charge qui pourrait se définir comme un barrage poids poreux. Il est constitué de deux murs minees divisant en trois parties la largeur, de profil triangulaire, et soutenant des barreaux horizontaux. Ces barreaux traversent toute la passe et sont scellés de part et d'autre dans les bajoyers (fig, 2 et 3). Le haut de cette grille épaisse se trouve dans la solution définitive à la cote 191 , donc à 7 ou $8 \mathrm{~m}$ sous le plan d'eau normal aval. Les barreaux ont un diamètre de $0,30 \mathrm{~m}$. On prévoit de les réaliser en tubes d'acier, emplis de béton alin d'augmenter leur inerlie. Des tirants appuieront les murs intermédiaires et l'ensemble de l'ouvrage sur le béton du radier qui, de toutes façons, est très épais en eet endroit.

On voit aisément que, dans un te! cadre, on dispose pour influencer l'écoulement d'un nombre considérable de degrés de liberté : on agira tuant le ressaut, le dénoyage du loil recouvrant les passes, el la difrusion laterale des veines liquides à la sortie.

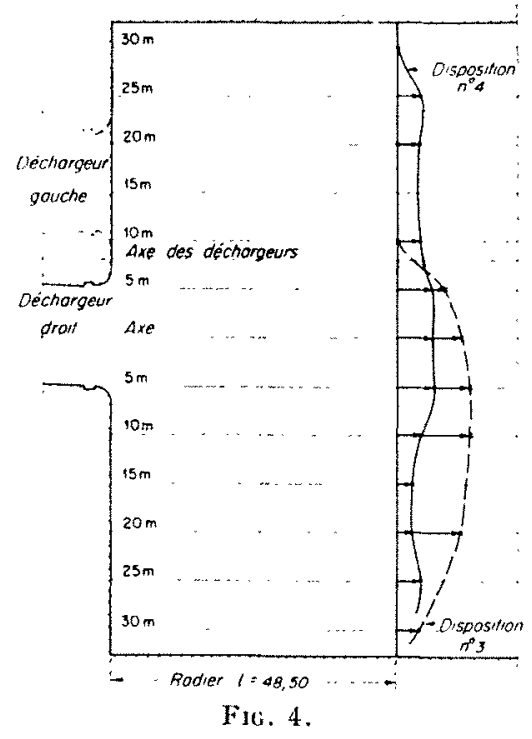

Ce dernier point mérite une mention particulière. Nous avons en effet constaté que si la grille n'est pas trop dense, une surélévation locale asse\% 


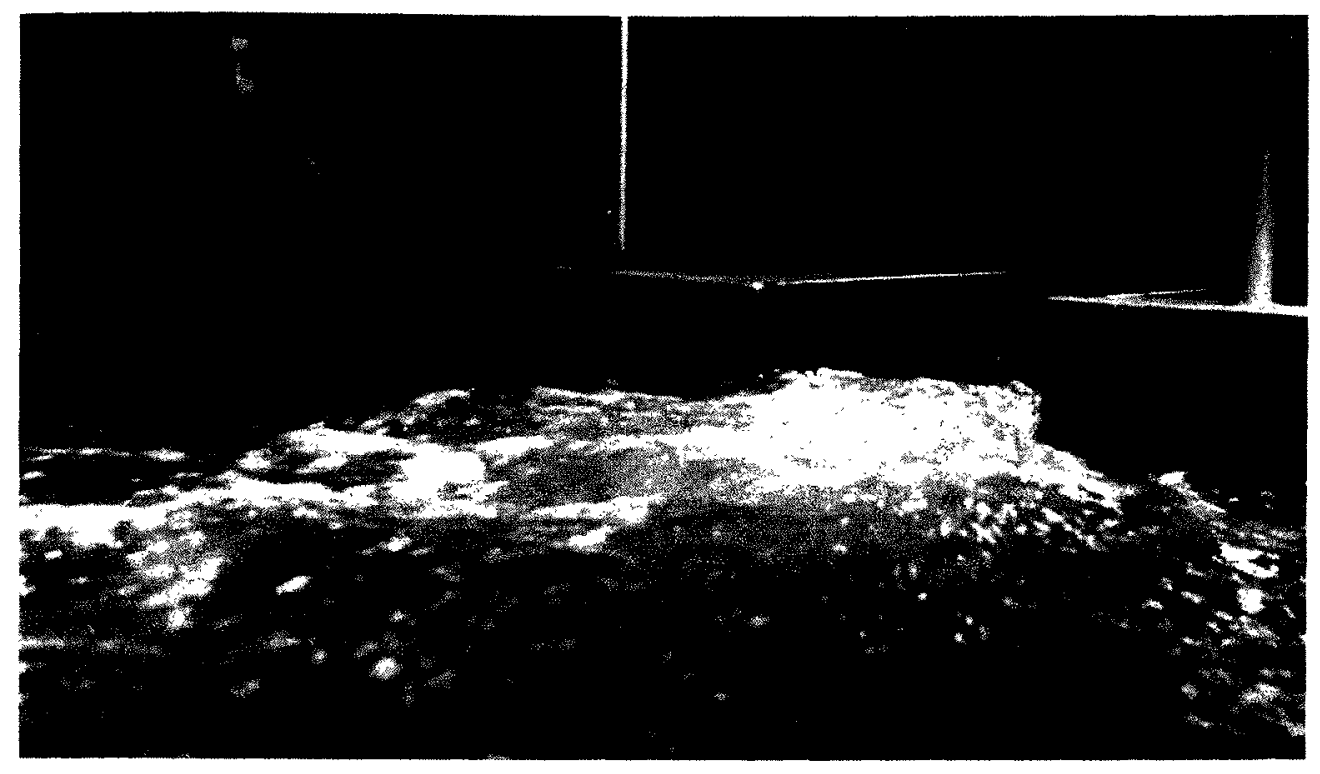

FIG. 5.
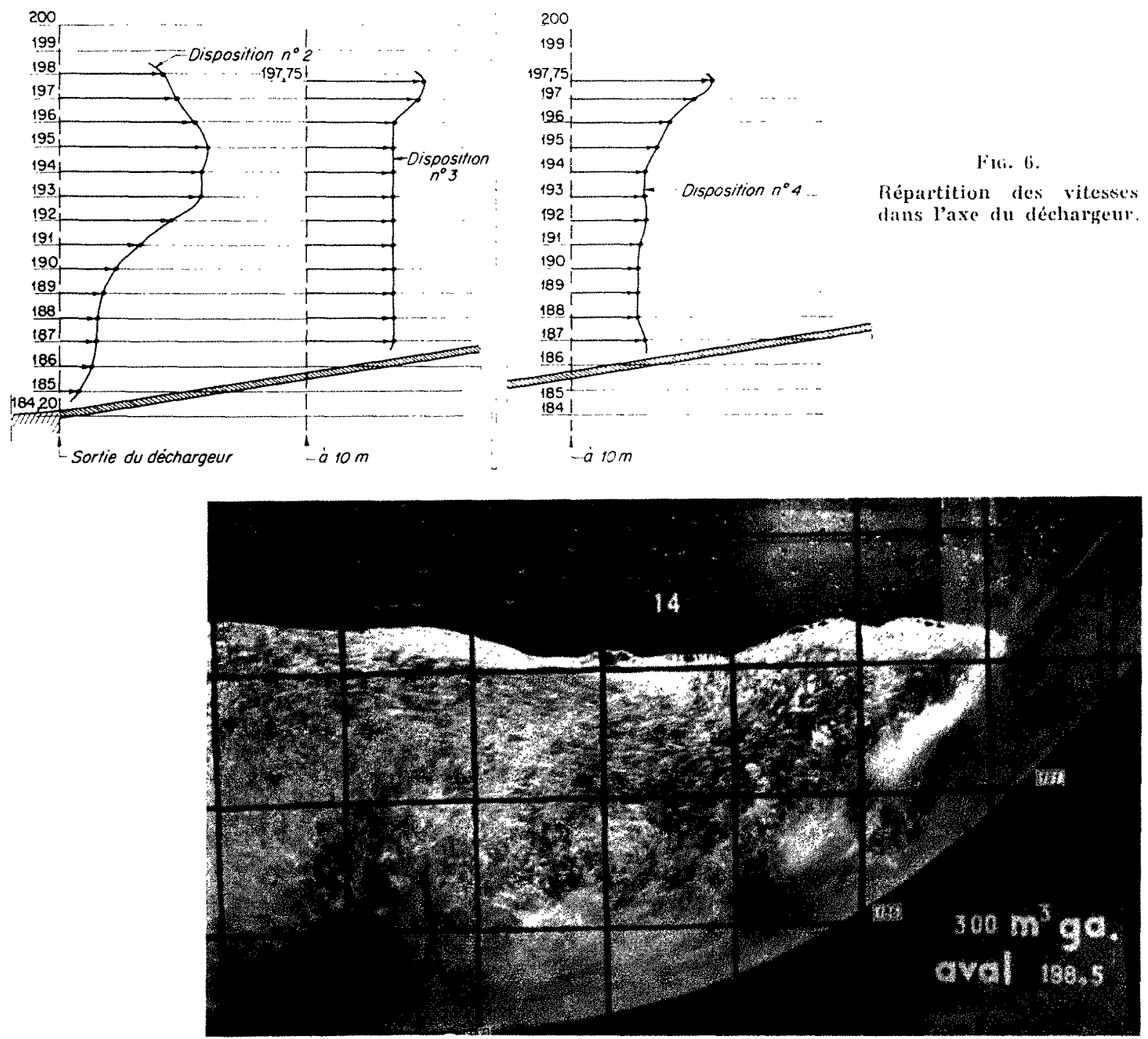

Fric 7 
importante et stable prend place un peu en aval du sommet du hrise-charge. Celui-ci étant placé le plus à laval possible dans les passes, dégageant juste les rainures de batardeaux, cette surélévation est cause d'une diffusion latérale importante de la veine. diffusion dont le demi-angle d'ouverture atteint, en surface du moins. 40 à 15) degrés (fig. 1 et 5 ).

En ce qui concerne la répartition verticale des vilesses, il chail farile d'obtenir par ajustements successifs un compromis conduisant à la limile de stabilité des malériaux de fond et à une am. plitude acceptable des perturbations de surface (fig. 6).

La visualisation les lignes de comrant dans le rouleau horizontal lic au ressaut et à travers la grille permettait a l'intuition de jouer un grand ròle dans le perfectionnement du dispositif ifig. 7 ).

\section{III}

Lorsyue nous avons envisage la mise en place d'un tel ouvrage, nous avons évidemmenl éprouve la crainte de vibrations importantes engendrées par l'écoulement. Il n'est pas impensable que de telles vibrations, si elles correspondaient à un régime de résonance entre la fréquence de détachement des tourbillons el des lluctuations de pressions qui lui sont liées et la lréquence propre des barreaux, puissent causer des ruptures rapides.

Les conditions de similitude de ces phénomines, malgré certaines tentatives, sont encole trop) mal définies pour que l'on puisse accorder sur ce point une confiance suffisante à un modele réduil. Nous avons préféré une expérience en varaic grandeur, pensant que les approximations faites seraient moins inquiétantes que l'adoption d'hypothéses douteuses nécessaire" à une étude sur modele. Ces essais furent menés par la Division Essais Extérieurs, sous la direction de M. Laterrier. Un barreau analogue à ceux pró. vus fut installe an bas du coursier de vidange d'une usine hydraulique où là veine présentait triss sensiblement les mêmes caractéristiques que celles prévues à Fessenheim, en w qui concernc ses dimensions ef sa vitesse. I.e barreau représenté ne correspondait qu'à un ćlément constiluant le liers de la largeur totale d'une passe. Ifin de tenir compte de la mauvase définition des conditions d'encastrement, le barreau étail fixé à chaque extrémité à une chaise par trois collier's : deux de ces colliers pouvant être supprimés, les conditions pouvaient-être modifiées sensiblement depuis celles d'un encastrement pur à celles d'un appui simple. Le barreau, en lube d'acier, pouvait être ou non empli d'cau, son inertie ćtant ainsi modifiéc. La fréquence propre du barreau ressortait, d'après le calcul, a un chifre compris entre 100 ef 200 p.s. selon les conditions de fixation.

Ces essais permirent de mettre en lumirre deux domaines de vibrations distincts :

a) Des vibrations à fréquence élevèc $(150 \mathrm{c} / \mathrm{s}$ environ) correspondant a la vibration propre du barreau. Les ampliludes de ces vibrations claient faibles el conduisaient it des contraintes maxima de l'ordre de $0,1 \mathrm{~kg} \mathrm{~m} / \mathrm{m}^{2}$;

b) Des vibrations à frequence heaucoup plus bas$\operatorname{ses}(8 \mathrm{c} / \mathrm{s})$, ivec une amplitude de 2 à $4 \mathrm{~m}$ pour la variation de pression dynamique et de 0,3 à $0,5 \mathrm{~kg} \mathrm{~m} / \mathrm{m}^{\prime}$ pour la variation des contraintes dans le métal. Il s'agit là de vibrations forcées dues à l'écoulemen! lui-même. La grande différence des fróquences observées et les efforts faibles mis en jeu rendent peu probables des phinomines dangereux de résonanre.

\section{VIII}

La présence de deux usines amont et la nature du terrain et de la végétation le long du canal rendent bien peu probable l'arrivé à Fessenheim de troncs d'arbres ou de branchages.

D'autre part, le fait que $8 \mathrm{~m}$ d'eau libre se Irouvent au-dessus de l'ouvrage et l'inclinaison des faces amont des murs intermédiaires sont de nature a en faciliter le passage.

Enfin, l'ćventualité d'une obstruction partielle ne présculerail cerlaimement pas le caractère d'une catastrophe.

\section{IX}

Les techniciens de laboratoire connaissenl déjà bien le moyen de réduire la longueur des canaux d'essais en y établissant dès l'entrée unc répartition de vitesse voulue a priori. Ils emploient pour cela des grilles épaisses dont on lait rarier la densité jusqu’à l'obtention du régime désiré.

Le dispositif mis au point par Fessemheim nest qu'une transposition de cet «accessoire». Son trait le plus marquant est son extrême souplesse d'adaptation, qui lui confère un intérêt assez général. 


\section{DIS CUSSION}

II. W Président Hopsen souligne le trés grand intéret de la communication de M. BRATUEAV et prie M. LefoUtos, Directeur de la Région d'Equipement Hydraulique Nord a E.D.F. de bien vouloir commenter l'expose de i. Bracbeal at préciser les différences que présente le dispositif d'amortissement employé a ottmarsheim avec celui qui a cle atopté pour fessenheim, ainsi que les misons qui lont conduit à cette décision.

M. Leforcos fait lexpose suivant:

" Deux raisons nous ont conduits a rechercher un dispositif different dottmarsheim :

l'abord une dronomie du génie civil:

Lutilisation de toute la largeur du canal, comme la fort bien dit M. Bratreat, conduil a réalisel des decharges de l'orde de $60 \mathrm{~m}^{3}$ par l'ouverlure de elapets ayant chacun un organe de manoure particulier.

La multiplicite de ces organes (24) nécessite une verbitable salle des machines qui oceupe tont le mur amont.

D'autre part, les déchargeurs eux-mémes accupent tout If volume séparant les groupes. Les coursiers entourant chaque groupe, au nombre de six, ont éle l'objet d'études sur modele réduit a Chatou; leur exécution réclame une construction en béton armé délicate al coùtense.

Dire que cette conception de déchargenrs, qui débouchent au-dessus de's bâches des aspirateurs de chaque groupe, correspond a la solution la meilleure pour dissiper l'énergic at que les résultats obtenus en réalite ont confirmé ceux sur modèle. cela semble bien diffeile. Pendant la décharge de 1.000 à $1.100 \mathrm{~m}:$ qui a été op:ré pendant plus de six mois en attendant la mise eu route du premier groupe, on a constaté bien des remous. bien des tourbillons, dans les coursiers et sur les doucines, Les intumescences de surface étaient importantes, mais heureusement s'amortissaient suffisamment lorsqu'elles arrivaient dans le canal de navigation. Cependant, nous avons été obligés de rebétonner les doucines dont les fers avaient été mis à nu par la violence des tourbillons. Il faut reconnaitre que les déchargeurs ont eté soumis a une épreuve exceptionnelle qu'ils ac comaitront plus à l'arenir. C'est considérable $1.000 \mathrm{~m}$ : pendant six mois!

Remarquons que dans le dispositif d'ottmarsheim la lurbulence dans le canal de fuite n'intéresse que la couche d'eau en surface et que le fond du canal de fuite n'a pas a la moindre irosion.

Aussi, nous arons pensé que, techniquement, nous arriverions i trouver une solution au moins aussi salisfaisante tout en cherchant ì réaliser, non seulement une economie de génic civil, mais également une économir de matériels mécaniques. Il faut bien reconnaître qu'un dispositif comprenant 4 vannes secteurs de $4 \times 6 \mathrm{~m}$ représente une dépense d'achat et d'installation moindre que celle de 24 clapets.

M. Bhatovar n'a peutêtre pas insisté sur l'amelioration que le nouveau type de dechargeur apportera dans la diminution de l'élongation des intumescenes. Comme nous l'a fort bien indiqué M. Bratdeal, dans l'installation des déchargeurs, on poursuit un double but :

1" Supprimer les intumescences positives et négatives a l'amont ef a l'aval dans le canal au moment d'une decharge brusque;

2" Eviter un abaissement subil du plan deau aval dams le garage aval.

Des essais faits les 2 mars et 15 mai $194 \overline{7}$, par la Division des Essais Exterieum des Etudes of Recherches
d'F.l. F. sue le bief do kembs (1) ont montre que lit coupure brusque de la charge de l'usine provoquait une intumeseence de $80 \mathrm{~cm}$ - celte onde remontant le ranal de navigation. - Si les chalands et automotenrs peurent fateilement supporter des ondes de 30 is $40 \mathrm{~cm}$, une ando de $80 \mathrm{~cm}$ peut provoquer des avaries ou des incidents.

Pour supprimer on atténuer l'intumeseence il faut pro voquer le plus rapidement possible te rétablissement da débit. Voici l'idée que nous arons ene :

Cne turbine Kaplan du trpe Fessenheim aura des servo-moteurs qui termeront le distribuleur d: la position ouverture pleine charge a la position marche a vide en quatre secondes l... Si nous associons a cette turbine un organe de décharge qui sera commande par un servo-moleur de mème nature mais plus puissant, nous pourroms essayer d'oblenir, en mème temps qu'une simultanéité de commande, nne simultanété de formelure el d'ouverture des débits.

"Je dessine le diagramme de fermeture d'une turbino lont le débit passe de $290 \mathrm{~m}:$ is $20 \mathrm{~m}:$ (débit a vide) en t secondes 1. Je dessine le diagrammo d'ouverture d'uno vanne de déchargeur eapable de latisser passer $290 \mathrm{~m}^{3}$. dans te cas d'une décharge brusque par la disjonetion du groupe. La disjonction commande simultanement la fermeture de la turbine et l'ouverture de la vanne. lin

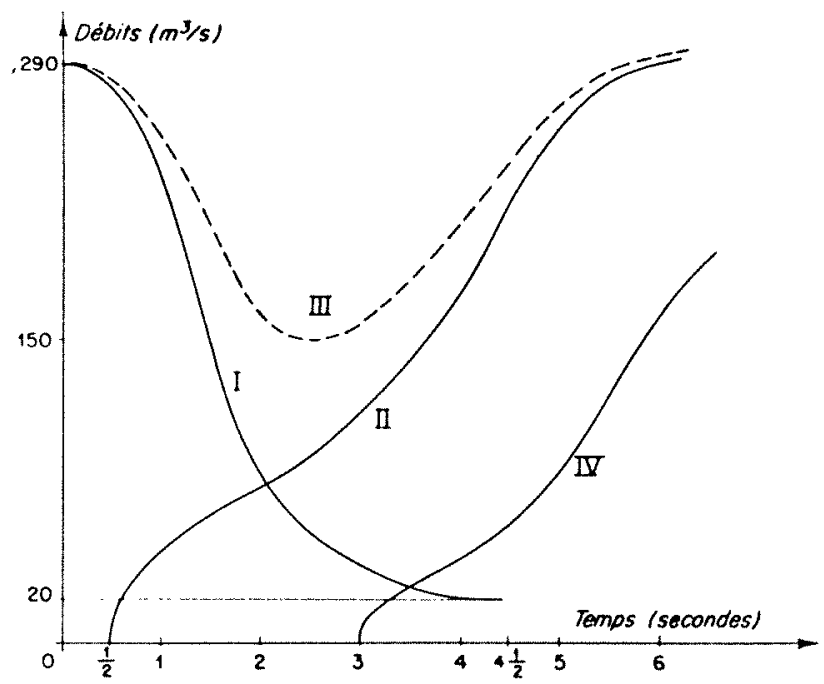

I. -... Fermelure de la turbine.

11. - Ouverture de la vame de decharge teas de disjonction is l'usine.

III. - Débit résultant.

IV. Ouverture de la vamue de dicharge feas de disjonction lointaine).

admettant unc constante de lemps de $1 / 2$ seconde dans la transmission de la commande, le diagramme d'ouver. ture en six secondes est celui indiqué. La débit résultant dans le canal est donné par la courbe pointillée. l.e débit minimum sera de l'ordre de $150 \mathrm{~m}^{3}$, peut-être $160 \mathrm{~m}^{*}$ et l'intumesence sera alors reduite de moitie et

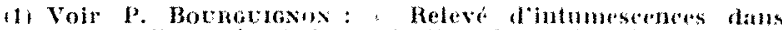
les ouvrages d"amenéc et de restitution de lusinw do fembs. Genzoires ef Traboux, de la S.H.F. Paris, 1948 , no 2 
de lorde de 30 a $40 \mathrm{~cm}$. c'est-à-dire tout is fait alceptable pour la navigation.

Nous pourons dire que ce cas se produira à chaque disjonction a lusine telepart du groupe) ce qui est to cas grave. Si nous sommes en presence d'une disparilion de la charge, par un affaissement du resean a la suite d'une disjonction lointaine', nous n'aurons plus la possibilité d'une commande immédiate de l'ouverture de la vanne par la disjonetion d'un disjoncteur, mais nows lobtiendrons par un relais assujetti a cercle de vannage de la turbine.

Sans attendre 4 secondes $1 / 2$, dure de fermeture de la turbine, je propose que lorsque la charge aura disparu des $2 / 3$ de sa valeur, louverture de la vanne de decharge soit commande. Cela se passera au bout de Irois secondes. Le debit sera reduit it 80 ou $100 \mathrm{m:}$ vuis remontera, le diagramme d'ouverture se faisant suivant Ia courbe IV corigine i 3 secondes).

Jestime que lorsqu'une usine comme Fessonheim, qui doit produire de l'energic de hase et qui debitera sur un résea bouclé à $220 \mathrm{kV}^{r}$ ou a $150 \mathrm{kV}$, aura un abaicsement de sa puissance des $2 / 3$ pour fixer les idées, e'est que la charge disparaitra totalement et il n'est pas besoin d'attendre que la lurbine soit à la marche ì ride pour ouvrir la vanue de décharge. Nous obtiendrons donc, dans ce cas, une réduction moins importante de l'intumescence, mais toutefois une reluction interessante. Or, d'aprés nous, ee cas doit être assez rare.

Enfin, le rétablissement du débit a l'aral doit éviter. un second danger : Pabaissement du plan d'eau dans le garage aval des éluses et même dans le cours du Rhin i l'aval. Le déchargeur prévu est assez rapide pour éviler tout abaissement du plan d'eau à l'aval.

Ponr terminer, nous arons voulu, en adoptant le nou reau dispositif pour les déchargeurs de Fessenheim, remplir notre rôle d'Ingénieur de l'Equipement qui ne consiste pas uniquement à résoudre une question par une belle reussite technique, mais a obtenir te même résullat le plus économiquement possible. Or, nous espérons gagner sur le matériel électro-mécanique quelques centaines de millions et sur le génie eivil $60.000 \mathrm{~m}^{3}$ de béton armé.

M. le President remercie vivement M. LkForLox de son intervention qui montre très clatrement comment se situe le probleme et son importance. Il rappelle que, daus certains dépaltements, des arrêtés préfectoraux datant du Second Empire el reglementamt les moulins, astreignaient les meuniers a relier mécaniquement les vames des roues a aube a celles d'un «dechargeoir», afin, en eas de diminution de charge de la rouc, d'assurer la constance du débit transmis et d'éviter les int umescences.

M. Menot ajoute que des preseriplions amalogues existaient dans certains Cahiers des Charges d'usines du Massif Contral, il $y$ a cinquante ans.

M. Remendenas croit que le danger des intumescences positives pour la navigation réside moins dans leur hauteur que dans la pente de leur tète. Du fail de son inertie le bateau risque de ne pas suire assez vite la montée du plan d'eau. Mais en raison de la faible pente de la tête de l'intumescence. ce risque est faible en général. Dans l'exemple des fermetures de Fessenheim. le batea aura à franchir une dénivellation de $0,80 \mathrm{~m}$ de hatut en 4 secondes $1 / 2$, e'est-à-dire sur une longueur de $50 \mathrm{~m}$ (célérités de l'ordre de 10 it $12 \mathrm{~m} / \mathrm{s}$ ); on voit quo la pente de la tète de l'intumescence n'est que de $1,6 \%$.

I. Bratomal confirme que ce que l'ou craint e'est surtout l'intumesernce négative, e'est-itdire la baisse du plan d'ean qui suit la première onde et qui, sur le Rhin, met certains bateaux en danger de talonnage.
M. Mesot signale que te probleme est resolu pour la Seine Maritime oú au moment de l'artivé du flof. les bateaux s'en ront foujours an milieu pour avoir une intumescence minimum et ne restent pas sur les rives, ou le flot atteint sa puissance maximum.

Répondant a plusieurs questions successives de M. la president, M. Bhat'oner indique :

1" Que Ies mesures faites sur des barreaux ch gralldeur nature, it l'usine de Vaufey, au moyen d'ildicateurs de pression, ont permis de distinguer très nettement deux genres de vibrations peu influencées, surtout les secondes, pill les conditions d'encastrement el l'obliquile des harreanx :

a) une vibration de periode comprise entre $120 \mathrm{et}$ 160 cycles par seconde el correspondant sensiblement ì la période propre des barreaus détermince par le calcul, soit 150 cycles,

b) une vibralion a basse fréguence de lordre de 8 eveles par seconde qu'on a alleibue anx phénomènes hydrauliques.

2" Que, pour les rannes, la separation des vibrations est possible si la période propre de cet organe et la pseudo période des phénomènes hydrauliques sont extrêmement différentes; toutefois, lorsque ees périodes sont voisines, et e'est précisement és caslit qui sont dangereux, la séparation es( beauconp, plus difficile.

D'ailleurs, le probleme de la vibration des organes hydrauliques est généralement lres difficile à étudier sir modèle, soit parce que l'on connait très mal les conditions de similitude mécanique des phénomènes périodiques d'origine hydraulique, soil que l'on déduise wes souvent de ce que l'on connat de cette similitude l'impossibilité de procéder à une élude sur modèle par suite d'une trop grande exiguilé de la bande du nombre de Reymolds dont on dispose.

M. Gumbe indique, en réponse à II. Io president, que l'examen des ribrations anormales de certaines passes du barage de Chatou, a du ctre retardé jusqu'à es gu'un entretien general des vannes soit engage parce que l'iustallation sur te barrige des prises de pression nécessaires à ect examen implique la mise en place des balardeanx.

M. Drelwaxos, sollicile par M. Le Président, dit que les experiences analogues prevues sul le barrage de Kerrata nont pas encore dé fajtes.

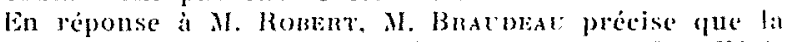
réduction de jechelle de la lurbulence al pour effet d'éviter une trop nefte localisalion de la dissipation d'energie, cause de dioridations importantes. La dissipalion se fait, comme dans tous les cas, par frottements sur le fond ef par turbulence au sein du flude, mais les frottements sont dissemines sur toute la surface de la paroi et en aucun poinl la densité de l'énergic dissipée n'est suffisante pour causer des degradations importantes du fond (effet de pomme d'arrosoir, comme lindique M. Romsirr).

M. Ronerr indique qu'il a utilise cet effet, en tant qu'Ingenieur des Ponts "l Chaussces, pour realiser au pied d'un aquedue, un bassin de decantation ou de franquillisation destinè a transformer en cals dorage, un flol très rapide en un flot résulier : l'effot a bi oblenu en faisant passer le flot à travers un écran en béton percé de trous. M. Bratuead confirme que le principe de ce dispositif est le même que celui qui sera utilisé pour les dechargeurs de Fessenheim.

M. Levorrox ajoute : 
Le nombre de barraux a été considerablement diminué et le résultat oblenu tient plus à la répartition des barreaux qu'a leur nombre. Si nous n'avions pas ce dispositif dans la chambre de décharge où nous ayous une hauteur d'eau de $15 \mathrm{~m}$, le rouleau qui se forme dans la chambre se ferait dans le canal de fuite a l'aval de l'usine.

"J'ajoute que le probleme était particulierement difficile à Fessenheim où nous arons une usine fondé sur $200 \mathrm{~m}$ d'alluvions. Il fallait que la decharge se fasse. non seulement sans intumescence de surface, mais également sans affouillement à l'aval pour ne pas dechausser le radier aval de l'usine.

« M. Braudeat a fait des essais fres inleressants de decharge sans le disposilif a barreaux el on a vu l'im- porlance des affouillements a laval. La question sost du reste compliquée par limportance de la pente des aspiratenrs des turbines: $16 \%$ - pente que, pour des masons de genie eivil, nous avons adoplé pour laval du dechirgeur.

« Actuellement nous etudions la nature du revalement des quelque $150 \mathrm{~m}$ a l'aval du eamal te fuile. Aprés $30 \mathrm{~m}$ de radier betonne il semble indique de tapisser le canal par des entochements et graviers a granula métrie decroissante pour éviter le moindre affouillement. s

M. Le President remereic M. Bhacmbat et souligme que le nombre des questions posces montre fout l'intéret de cette communication.

\section{AVIS DE CONCOURS}

Un concours est ouvert en vue :

1" De l'étude, de la construction et de la fourniture a pied d'ouvre du matériel destiné á régler la ligne d'eau du canal principal d'irrigiation de la plaine des Triffa (Maroc Oriental) et le débit des prises des canaux secondaires piqués sur ce canal;

2" Du montage des régulateurs automatiques, faisant partic du matericl susvisé.

Le cautionnement provisoire est fixé it 1 million de francs.

Le dossier complet du concours peut itre consulte :

- dans les bureaux de la circonscription de l'Hydraulique et de l'Electricité à RABAT;

-. dans les bureaux de la Subdivision hydraulique a BERKaxe;

.... a l'Office du Maroc, 19, rue des Pyramides à Paris.

Cn dossier restreint est mis a la disposition des entrepreneurs dans les burcaux de l'Office du Maroc à :

- Tyon, Chambre de Commerce, rue de la République;

-... Bordeaux, place Cabriel:

- Marseille, 2, me Beauvau:

Strasbourg, Chambre de Commerce, place Gulenbers:

lille. 116, rue de l'Hopital-Mililaire:

Naney, chey M. Brun. 10, rue Gambetta.

La date limite de remise des offres date de dépot dans les bureaus de la circonseription de l'hydraulique ef de lélectricite a Rabat ou de remise a la poste sous pli recommande - avionl est fixere an 28 arril 1955 avant 12 houres. 